\title{
The $W_{2}$-curvature tensor on warped product manifolds and applications
}

\author{
Sameh Shenawy \\ Basic Science Department \\ Modern Academy for Engineering and Technology \\ Maadi, Egypt \\ drssshenawy@eng.modern-academy.edu.eg; \\ drshenawy@mail.com \\ Bülent Ünal \\ Department of Mathematics \\ Bilkent University, Bilkent, 06800 Ankara, Turkey \\ bulentunal@mail.com
}

Received 2 August 2015

Accepted 27 April 2016

Published 7 June 2016

\begin{abstract}
The purpose of this paper is to study the $W_{2}$-curvature tensor on (singly) warped product manifolds as well as on generalized Robertson-Walker and standard static space-times. Some different expressions of the $W_{2}$-curvature tensor on a warped product manifold in terms of its relation with $W_{2}$-curvature tensor on the base and fiber manifolds are obtained. Furthermore, we investigate $W_{2}$-curvature flat warped product manifolds. Many interesting results describing the geometry of the base and fiber manifolds of a $W_{2}$-curvature flat warped product manifold are derived. Finally, we study the $W_{2}$ curvature tensor on generalized Robertson-Walker and standard static space-times; we explore the geometry of the fiber of these warped product space-time models that are $W_{2}$-curvature flat.
\end{abstract}

Keywords: $W_{2}$-curvature; standard static space-time; generalized Robertson-Walker space-time; warped products.

Mathematics Subject Classification 2010: 53C21, 53C25, 53C50

\section{Introduction}

In [1], Pokhariyal and Mishra first defined the $W_{2}$-curvature tensor and they studied its physical and geometrical properties. Since then the concept of the $W_{2}$-curvature tensor has been studied as a research topic by mathematicians and physicists (see $[2-5])$. Pokhariyal defined many symmetric and skew-symmetric curvature tensors on the same line of the $W_{2}$-curvature tensor and studied various geometrical and physical properties of manifolds admitting these tensors in [3]. Among many of his results, we would like to mention that he proved that the vanishing of one of 
these curvature tensors in an electromagnetic field implies a purely electric field. Another study to establish applications of the $W_{2}$-curvature in the theory of general relativity was carried in [6] where the authors particularly prove that a space-time with vanishing $W_{2}$-curvature tensor is an Einstein manifold. They also consider the case of vanishing $W_{2}$-curvature tensor in relation with a perfect fluid space-time. In $[2,5]$, the authors study the properties of flat space-time under some conditions regarding the $W_{2}$-curvature tensor and $W_{2}$-flat space-times. Moreover, there are many studies regarding the geometrical meaning of the $W_{2}$-curvature tensor in different types of manifolds (see [7-10] and references therein).

The main aim of this paper is to study and explore the $W_{2}$-curvature tensor on warped product manifolds as well as on well-known warped product space-times. The concept of the $W_{2}$-curvature tensor has never been studied on warped products before this paper in which we intent to fill this gap in the literature by providing a complete study of the $W_{2}$-curvature tensor on such spaces.

This paper is organized as follows. In Sec. 2, we state well-known curvature related formulas of warped product manifolds and the $W_{2}$-curvature tensor properties on pseudo-Riemannian manifolds. We also define and study a new curvature tensor, $K(X, Y) Z$, that will be used in the characterization of the $W_{2}$-curvature tensor on pseudo-Riemannian manifolds. In Sec. 3, we explore the relation between the $W_{2}$-curvature tensor of a warped product manifold and that of the fiber and base manifolds. Section 4 is devoted to the study of the $W_{2}$-curvature tensor on generalized Robertson-Walker space-time and standard static space-time.

\section{Preliminaries}

In this section, we will provide basic definitions and curvature formulas about warped product manifolds.

Suppose that $\left(M_{1}, g_{1}, D_{1}\right)$ and $\left(M_{2}, g_{2}, D_{2}\right)$ are two $\mathcal{C}^{\infty}$-pseudo-Riemannian manifolds equipped with pseudo-Riemannian metric tensors $g_{i}$ where $D_{i}$ is the Levi-Civita connection of the metric $g_{i}$ for $i=1,2$. Further suppose that $\pi_{1}$ : $M_{1} \times M_{2} \rightarrow M_{1}$ and $\pi_{2}: M_{1} \times M_{2} \rightarrow M_{2}$ are the natural projection maps of the Cartesian product $M_{1} \times M_{2}$ onto $M_{1}$ and $M_{2}$, respectively. If $f: M_{1} \rightarrow(0, \infty)$ is a positive real-valued smooth function, then the warped product manifold $M_{1} \times_{f} M_{2}$ is the product manifold $M_{1} \times M_{2}$ equipped with the metric tensor $g=g_{1} \oplus f^{2} g_{2}$ defined by

$$
g=\pi_{1}^{*}\left(g_{1}\right) \oplus\left(f \circ \pi_{1}\right)^{2} \pi_{2}^{*}\left(g_{2}\right),
$$

where $^{*}$ denotes the pull-back operator on tensors $[11,12]$. The function $f$ is called the warping function of the warped product manifold $M_{1} \times_{f} M_{2}$. In particular, if $f=1$, then $M_{1} \times{ }_{1} M_{2}=M_{1} \times M_{2}$ is the usual Cartesian product manifold. It is clear that the submanifold $M_{1} \times\{q\}$ is isometric to $M_{1}$ for every $q \in M_{2}$. Moreover, $\{p\} \times M_{2}$ is homothetic to $M_{2}$. Throughout this paper we use the same notation for a vector field and for its lift to the product manifold. Let $D, R$ and Ric be the 
Levi-Civita connection, curvature tensor and Ricci curvature of the metric tensor $g$. Their formulas are well-known (see $[11,12])$.

The $W_{2}$-curvature tensor on a pseudo-Riemannian manifold $(M, g, D)$ is defined as follows [1]. Let $X, Y, Z, T \in \mathfrak{X}(M)$, then

$$
\begin{aligned}
W_{2}(X, Y, Z, T)= & g(R(X, Y) Z, T) \\
& +\frac{1}{n-1}[g(X, Z) \operatorname{Ric}(Y, T)-g(Y, Z) \operatorname{Ric}(X, T)],
\end{aligned}
$$

where $R(X, Y) Z=D_{Y} D_{X} Z-D_{X} D_{Y} Z+D_{[X, Y]} Z$ is the Riemann curvature tensor. It is clear that $W_{2}(X, Y, Z, T)$ is skew-symmetric in the first two positions. More explicitly, $W_{2}(X, Y, Z, T)=-W_{2}(Y, X, Z, T)$.

Now we redefine $W_{2}$-curvature tensor as follows. The $W_{2}$-curvature tensor, as shown above, is also given by

$$
W_{2}(X, Y, Z, T)=g(K(X, Y) T, Z),
$$

where

$$
K(X, Y) T:=-R(X, Y) T+\frac{1}{n-1}[\operatorname{Ric}(Y, T) X-\operatorname{Ric}(X, T) Y] .
$$

The study of the $W_{2}$-curvature tensor on warped product manifolds contains large formulas and a huge amount of computations. Thus, this new tool will enable us to minimize computations in our study.

Remark 1. Let $M$ be a pseudo-Riemannian manifold. Then

$$
K(X, Y) T+K(T, X) Y+K(Y, T) X=0
$$

for any vector fields $X, Y, T \in \mathfrak{X}(M)$.

The following proposition is a direct consequence of the new definition of the $W_{2}$-curvature tensor.

Proposition 2. Let $M$ be a pseudo-Riemannian manifold. Then the $W_{2}$-curvature tensor vanishes if and only if the tensor $K$ vanishes.

Now, we will note that the tensor $K$ can be simplified if the last position is a concurrent field. First, recall that a vector field $\zeta$ is called a concurrent vector field if

$$
D_{X} \zeta=X
$$

for any vector field $X$. It is clear that a concurrent vector field is a conformal vector field with factor 2 . Let $\zeta$ be a concurrent vector field, then

$$
R(X, Y) \zeta=0 \text {. }
$$

Now suppose that $\zeta$ is a concurrent vector field. Then

$$
K(X, Y) \zeta=\frac{1}{n-1}[\operatorname{Ric}(Y, \zeta) X-\operatorname{Ric}(X, \zeta) Y]
$$

Finally, a Riemannian metric $g$ on a manifold $M$ is said to be of Hessian type metric if there are two smooth functions $k$ and $\sigma$ such that $H^{\sigma}=k g$ where 
$H^{\sigma}$ is the Hessian of $\sigma$. This topic is closely related to the research of Shima on Hessian manifolds (see $[13,14]$ ) and its extension to pseudo-Riemannian manifolds in $[15,16]$.

\section{3. $W_{2}$-Curvature Tensor on Warped Product Manifolds}

In this section, we provide an extensive study of $W_{2}$-curvature tensor on (singly) warped product manifolds. Throughout the section, $(M, g, D)$ is a (singly) warped product manifold of $\left(M_{i}, g_{i}, D_{i}\right), i=1,2$ with dimensions $n_{i} \neq 1$ where $n=n_{1}+$ $n_{2} . R, R^{i}$ denote the curvature tensor and Ric, $\operatorname{Ric}^{i}$ denote the Ricci curvature tensor on $M, M^{i}$, respectively. Moreover, $\nabla f$ denotes the gradient and $\Delta f$ denotes Laplacian of $f$ on $M_{1}$, and also the Hessian of $f$ on $M_{1}$ is denoted by $H^{f}$. The sharp of $f$ is given by $f^{\sharp}=f \Delta f+\left(n_{2}-1\right) g_{1}(\nabla f, \nabla f)$. Finally, $W_{2}$-curvature tensor and the tensor $K$ on $M$ and $M_{i}$ are denoted by $W_{2}, K$ and $W_{2}^{i}, K^{i}$, respectively for $i=1,2$.

The following theorem provides a full description of the $W_{2}$-curvature tensor on (singly) warped product manifolds. The proof contains long computations that can be done using previous results on warped product manifolds (see Appendix A).

Theorem 3. Let $M=M_{1} \times_{f} M_{2}$ be a singly warped product manifold with the metric tensor $g=g_{1} \oplus f^{2} g_{2}$. If $X_{i}, Y_{i}, T_{i} \in \mathfrak{X}\left(M_{i}\right)$ for $i=1,2$, then

$$
\begin{aligned}
K\left(X_{1}, Y_{1}\right) T_{1}= & K^{1}\left(X_{1}, Y_{1}\right) T_{1} \\
& -\frac{n_{2}}{(n-1)\left(n_{1}-1\right)}\left[\operatorname{Ric}^{1}\left(Y_{1}, T_{1}\right) X_{1}-\operatorname{Ric}^{1}\left(X_{1}, T_{1}\right) Y_{1}\right] \\
& -\frac{1}{n-1}\left[\frac{n_{2}}{f} H^{f}\left(Y_{1}, T_{1}\right) X_{1}-\frac{n_{2}}{f} H^{f}\left(X_{1}, T_{1}\right) Y_{1}\right], \\
K\left(X_{1}, Y_{1}\right) T_{2}= & K\left(X_{2}, Y_{2}\right) T_{1}=0, \\
K\left(X_{1}, Y_{2}\right) T_{1}= & -\left[\frac{1}{n-1} \operatorname{Ric}^{1}\left(X_{1}, T_{1}\right)-\frac{n+n_{2}-1}{(n-1) f} H^{f}\left(X_{1}, T_{1}\right)\right] Y_{2}, \\
K\left(X_{1}, Y_{2}\right) T_{2}= & -f g_{2}\left(Y_{2}, T_{2}\right) D_{X_{1}}^{1} \nabla f+\frac{1}{n-1} \operatorname{Ric}^{2}\left(Y_{2}, T_{2}\right) X_{1} \\
& -\frac{f^{\sharp}}{n-1} g_{2}\left(Y_{2}, T_{2}\right) X_{1}, \\
K\left(X_{2}, Y_{2}\right) T_{2}= & K^{2}\left(X_{2}, Y_{2}\right) T_{2} \\
& -\frac{n_{1}}{(n-1)\left(n_{2}-1\right)}\left[\operatorname{Ric}^{2}\left(Y_{2}, T_{2}\right) X_{2}-\operatorname{Ric}^{2}\left(X_{2}, T_{2}\right) Y_{2}\right] \\
& +\left(\|\nabla f\|_{1}^{2}+\frac{f^{\sharp}}{n-1}\right)\left[g_{2}\left(X_{2}, T_{2}\right) Y_{2}-g_{2}\left(Y_{2}, T_{2}\right) X_{2}\right] .
\end{aligned}
$$

In the following part we investigate the geometry of the base factor of the warped product when the product is $W_{2}$-curvature flat. 
Theorem 4. Let $M=M_{1} \times_{f} M_{2}$ be a $W_{2}$-curvature flat singly warped product manifold with the metric tensor $g=g_{1} \oplus f^{2} g_{2}$. Then

$$
W_{2}^{1}\left(X_{1}, Y_{1}, Z_{1}, T_{1}\right)=\frac{2 n_{2}}{\left(n_{1}-1\right) f}\left[H^{f}\left(Y_{1}, T_{1}\right) g_{1}\left(X_{1}, Z_{1}\right)-H^{f}\left(X_{1}, T_{1}\right) g_{1}\left(Y_{1}, Z_{1}\right)\right]
$$

for any vector fields $X_{1}, Y_{1}, Z_{1}, T_{1} \in \mathfrak{X}\left(M_{1}\right)$.

Proof. Suppose that $M$ is $W_{2}$-curvature flat. Then Eqs. (1) and (3) imply that

$$
\begin{aligned}
0= & K^{1}\left(X_{1}, Y_{1}\right) T_{1}-\frac{n_{2}}{(n-1)\left(n_{1}-1\right)}\left[\operatorname{Ric}^{1}\left(Y_{1}, T_{1}\right) X_{1}-\operatorname{Ric}^{1}\left(X_{1}, T_{1}\right) Y_{1}\right] \\
& -\frac{1}{n-1}\left[\frac{n_{2}}{f} H^{f}\left(Y_{1}, T_{1}\right) X_{1}-\frac{n_{2}}{f} H^{f}\left(X_{1}, T_{1}\right) Y_{1}\right], \\
0= & \frac{1}{n-1} \operatorname{Ric}^{1}\left(X_{1}, T_{1}\right)-\frac{n_{1}+2 n_{2}-1}{(n-1) f} H^{f}\left(X_{1}, T_{1}\right) .
\end{aligned}
$$

Now, from the second equation we have

$$
\operatorname{Ric}^{1}\left(X_{1}, T_{1}\right)=\frac{n_{1}+2 n_{2}-1}{f} H^{f}\left(X_{1}, T_{1}\right) .
$$

Using this identity in the first equation which eventually turns out to be:

$$
\begin{aligned}
K^{1}\left(X_{1}, Y_{1}\right) T_{1}= & \frac{n_{2}}{(n-1)\left(n_{1}-1\right)}\left[\frac{n_{1}+2 n_{2}-1}{f} H^{f}\left(Y_{1}, T_{1}\right) X_{1}\right. \\
& \left.-\frac{n_{1}+2 n_{2}-1}{f} H^{f}\left(X_{1}, T_{1}\right) Y_{1}\right] \\
& +\frac{n_{2}}{n-1}\left[\frac{1}{f} H^{f}\left(Y_{1}, T_{1}\right) X_{1}-\frac{1}{f} H^{f}\left(X_{1}, T_{1}\right) Y_{1}\right] \\
= & \frac{2 n_{2}^{2}}{(n-1)\left(n_{1}-1\right) f}\left[H^{f}\left(Y_{1}, T_{1}\right) X_{1}-H^{f}\left(X_{1}, T_{1}\right) Y_{1}\right] .
\end{aligned}
$$

Thus

$$
W_{2}^{1}\left(X_{1}, Y_{1}, Z_{1}, T_{1}\right)=\frac{2 n_{2}}{\left(n_{1}-1\right) f}\left[H^{f}\left(Y_{1}, T_{1}\right) g_{1}\left(X_{1}, Z_{1}\right)-H^{f}\left(X_{1}, T_{1}\right) g_{1}\left(Y_{1}, Z_{1}\right)\right] .
$$

Theorem 5. Let $M=M_{1} \times_{f} M_{2}$ be a $W_{2}$-curvature flat singly warped product manifold with the metric tensor $g=g_{1} \oplus f^{2} g_{2}$. Then:

(1) $M_{1}$ is $W_{2}$-curvature flat if and only if $H^{f}\left(X_{1}, Y_{1}\right)=0$ for any vector fields $X_{1}, Y_{1} \in \mathfrak{X}\left(M_{1}\right)$.

(2) the scalar curvature $S_{1}$ of $M_{1}$ is given by

$$
S_{1}=\frac{n_{1}+2 n_{2}-1}{f} \Delta f .
$$

(3) the scalar curvature of $M_{1}$ vanishes if $M_{1}$ is $W_{2}$-curvature flat. 
Proof. The proof just follows from Eqs. (6) and (7).

Now, we study the geometry of the fiber factor of a warped product admitting flat $W_{2}$-curvature.

Theorem 6. Let $M=M_{1} \times_{f} M_{2}$ be a singly warped product manifold with the metric tensor $g=g_{1} \oplus f^{2} g_{2}$. Assume that $f$ satisfies $H^{f}=0$. Then, $M$ is $W_{2}$ curvature flat if and only if both $M_{1}$ and $M_{2}$ are flat and $\nabla f=0$.

Proof. Suppose that $M$ is $W_{2}$-curvature flat, then $M_{1}$ is flat due to Eq. (7) and the first item of Theorem 5. Moreover, from Theorem 3 we have

$$
\begin{aligned}
0= & -f g_{2}\left(Y_{2}, T_{2}\right) D_{X_{1}}^{1} \nabla f+\frac{1}{n-1} \operatorname{Ric}^{2}\left(Y_{2}, T_{2}\right) X_{1}-\frac{f^{\sharp}}{n-1} g_{2}\left(Y_{2}, T_{2}\right) X_{1}, \\
0= & K^{2}\left(X_{2}, Y_{2}\right) T_{2}-\frac{n_{1}}{(n-1)\left(n_{2}-1\right)}\left[\operatorname{Ric}^{2}\left(Y_{2}, T_{2}\right) X_{2}-\operatorname{Ric}^{2}\left(X_{2}, T_{2}\right) Y_{2}\right] \\
& +\left(\|\nabla f\|_{1}^{2}+\frac{f^{\sharp}}{n-1}\right)\left[g_{2}\left(X_{2}, T_{2}\right) Y_{2}-g_{2}\left(Y_{2}, T_{2}\right) X_{2}\right] .
\end{aligned}
$$

Since $H^{f}\left(X_{1}, Y_{1}\right)=0$, the first equation becomes

$$
\operatorname{Ric}^{2}\left(Y_{2}, T_{2}\right)=f^{\sharp} g_{2}\left(Y_{2}, T_{2}\right),
$$

where $f^{\sharp}=f \Delta f+\left(n_{2}-1\right) g_{1}(\nabla f, \nabla f)=\left(n_{2}-1\right) c^{2}$ where $c^{2}=g_{1}(\nabla f, \nabla f)$, i.e. $M_{2}$ is Einstein with factor $\mu=\left(n_{2}-1\right) c^{2}$ and

$$
\operatorname{Ric}^{2}\left(Y_{2}, T_{2}\right)=\left(n_{2}-1\right) c^{2} g_{2}\left(Y_{2}, T_{2}\right) .
$$

The second equation becomes

$$
K^{2}\left(X_{2}, Y_{2}\right) T_{2}=\frac{2\left(n_{2}-1\right) c^{2}}{(n-1)}\left[g_{2}\left(Y_{2}, T_{2}\right) X_{2}-g_{2}\left(X_{2}, T_{2}\right) Y_{2}\right] .
$$

Thus the $W_{2}$-curvature tensor of $M_{2}$ is given by

$$
W_{2}^{2}\left(X_{2}, Y_{2}, Z_{2}, T_{2}\right)=\frac{2\left(n_{2}-1\right) c^{2}}{(n-1)}\left[g_{2}\left(Y_{2}, T_{2}\right) g_{2}\left(X_{2}, Z_{2}\right)-g_{2}\left(X_{2}, T_{2}\right) g_{2}\left(Y_{2}, Z_{2}\right)\right] \text {. }
$$

But

$$
\begin{aligned}
W_{2}^{2}\left(X_{2}, Y_{2}, Z_{2}, T_{2}\right)= & R^{2}\left(X_{2}, Y_{2}, Z_{2}, T_{2}\right) \\
& +\frac{1}{n_{2}-1}\left[g_{2}\left(X_{2}, Z_{2}\right) \operatorname{Ric}^{2}\left(Y_{2}, T_{2}\right)-g_{2}\left(Y_{2}, Z_{2}\right) \operatorname{Ric}^{2}\left(X_{2}, T_{2}\right)\right] \\
= & R^{2}\left(X_{2}, Y_{2}, Z_{2}, T_{2}\right) \\
& +c^{2}\left[g_{2}\left(X_{2}, Z_{2}\right) g_{2}\left(Y_{2}, T_{2}\right)-g_{2}\left(Y_{2}, Z_{2}\right) g_{2}\left(X_{2}, T_{2}\right)\right] .
\end{aligned}
$$

Therefore,

$R^{2}\left(X_{2}, Y_{2}, Z_{2}, T_{2}\right)=\frac{\left(n_{2}-n_{1}-1\right) c^{2}}{(n-1)}\left[g_{2}\left(X_{2}, Z_{2}\right) g_{2}\left(Y_{2}, T_{2}\right)-g_{2}\left(Y_{2}, Z_{2}\right) g_{2}\left(X_{2}, T_{2}\right)\right]$ 
i.e. $M_{2}$ has a constant sectional curvature

$$
\kappa_{2}=\frac{\left(n_{2}-n_{1}-1\right) c^{2}}{(n-1)} .
$$

But the Einstein factor should be $\left(n_{2}-1\right) \kappa_{2}$ and hence

$$
n_{1}\left(n_{2}-1\right) c^{2}=0 .
$$

Thus $M_{2}$ is flat. The converse is straightforward.

Theorem 7. Let $M=M_{1} \times_{f} M_{2}$ be a $W_{2}$-curvature flat singly warped product manifold with the metric tensor $g=g_{1} \oplus f^{2} g_{2}$. If $M_{2}$ is Ricci flat, then the $W_{2}$ curvature of $M_{2}$ is given by

$$
\begin{aligned}
& W_{2}^{2}\left(X_{2}, Y_{2}, T_{2}, Z_{2}\right) \\
& \quad=\left(\|\nabla f\|_{1}^{2}+\frac{f^{\sharp}}{n-1}\right)\left[g_{2}\left(X_{2}, T_{2}\right) g_{2}\left(Y_{2}, Z_{2}\right)-g_{2}\left(Y_{2}, T_{2}\right) g_{2}\left(X_{2}, Z_{2}\right)\right]
\end{aligned}
$$

and $M_{1}$ is of Hessian type. Moreover, $M_{2}$ is flat if $n_{2} \geq 3$.

Proof. Suppose that $M$ is $W_{2}$-curvature flat, then from Theorem 3 we have

$$
\begin{aligned}
0= & -f g_{2}\left(Y_{2}, T_{2}\right) D_{X_{1}}^{1} \nabla f+\frac{1}{n-1} \operatorname{Ric}^{2}\left(Y_{2}, T_{2}\right) X_{1}-\frac{f^{\sharp}}{n-1} g_{2}\left(Y_{2}, T_{2}\right) X_{1}, \\
0= & K^{2}\left(X_{2}, Y_{2}\right) T_{2}-\frac{n_{1}}{(n-1)\left(n_{2}-1\right)}\left[\operatorname{Ric}^{2}\left(Y_{2}, T_{2}\right) X_{2}-\operatorname{Ric}^{2}\left(X_{2}, T_{2}\right) Y_{2}\right] \\
& +\left(\|\nabla f\|_{1}^{2}+\frac{f^{\sharp}}{n-1}\right)\left[g_{2}\left(X_{2}, T_{2}\right) Y_{2}-g_{2}\left(Y_{2}, T_{2}\right) X_{2}\right] .
\end{aligned}
$$

Now suppose that $M_{2}$ is Ricci flat, then the first equation implies that

$$
D_{X_{1}}^{1} \nabla f=\frac{-f^{\sharp}}{(n-1) f} X_{1}
$$

and so

$$
H^{f}=\frac{-f^{\sharp}}{(n-1) f} g_{1},
$$

i.e. $M_{1}$ is of Hessian type. The second equation implies that

$$
K^{2}\left(X_{2}, Y_{2}\right) T_{2}=\left(\|\nabla f\|_{1}^{2}+\frac{f^{\sharp}}{n-1}\right)\left[g_{2}\left(X_{2}, T_{2}\right) Y_{2}-g_{2}\left(Y_{2}, T_{2}\right) X_{2}\right]
$$

and hence

$$
\begin{aligned}
& W_{2}^{2}\left(X_{2}, Y_{2}, T_{2}, Z_{2}\right) \\
& \quad=\left(\|\nabla f\|_{1}^{2}+\frac{f^{\sharp}}{n-1}\right)\left[g_{2}\left(X_{2}, T_{2}\right) g_{2}\left(Y_{2}, Z_{2}\right)-g_{2}\left(Y_{2}, T_{2}\right) g_{2}\left(X_{2}, Z_{2}\right)\right] .
\end{aligned}
$$


Moreover,

$$
\begin{aligned}
& R^{2}\left(X_{2}, Y_{2}, T_{2}, Z_{2}\right) \\
& \quad=\left(\|\nabla f\|_{1}^{2}+\frac{f^{\sharp}}{n-1}\right)\left[g_{2}\left(X_{2}, T_{2}\right) g_{2}\left(Y_{2}, Z_{2}\right)-g_{2}\left(Y_{2}, T_{2}\right) g_{2}\left(X_{2}, Z_{2}\right)\right] .
\end{aligned}
$$

Thus $M_{2}$ has a pointwise constant sectional curvature given by

$$
\kappa_{2}=\|\nabla f\|_{1}^{2}+\frac{f^{\sharp}}{n-1} .
$$

If $n_{2} \geq 3$, then by Schur's Lemma, $M_{2}$ has a vanishing constant sectional curvature $\kappa_{2}=0$ since $M_{2}$ is Ricci flat.

\section{4. $W_{2}$-Curvature on Space-Times}

The study of $W_{2}$-curvature tensor on space-times is of great interest since this concept provides an access to several geometrical and physical properties of spacetimes. Among such applications, we want to mention that a $W_{2}$-curvature flat 4 dimensional space-time is an Einstein manifold [2, 5]. This section is subsequently devoted to the study of the $W_{2}$-curvature tensor on generalized Robertson-Walker space-times and standard static space-times. We will first consider some classical space-times. Obtaining the $W_{2}$-curvature tensor for these space-times contains long computations, and hence we omitted them.

- The Minkowski space-time is $W_{2}$-curvature flat since it is flat.

- The Friedman-Robertson-Walker with metric

$$
d s^{2}=-c^{2} d t^{2}+a(t)\left[\frac{d \eta^{2}}{1-k \eta^{2}}+r^{2}\left(d \theta^{2}+\sin ^{2} \theta d \phi^{2}\right)\right]
$$

is $W_{2}$-curvature flat if $\dot{a}(t)=k=0$.

- The de Sitter space-time metric with cosmological constant $\Lambda>0$ in conformally flat coordinates reads

$$
d s^{2}=\frac{\alpha^{2}}{\tau^{2}}\left[-d \tau^{2}+d r^{2}+r^{2}\left(d \theta^{2}+\sin ^{2} \theta d \phi^{2}\right)\right],
$$

where $\alpha^{2}=(3 / \Lambda)$. This metric is Einstein with factor $\frac{3}{\alpha^{2}}$ and has a constant sectional curvature $\frac{1}{\alpha^{2}}$. The non-vanishing components of the $W_{2}$-curvature tensor are

$$
\begin{aligned}
W_{2}\left(\partial_{i}, \partial_{j}, \partial_{i}, \partial_{j}\right) & =R\left(\partial_{i}, \partial_{j}, \partial_{i}, \partial_{j}\right)+\frac{1}{3}\left(g\left(\partial_{i}, \partial_{i}\right) \operatorname{Ric}\left(\partial_{j}, \partial_{j}\right)\right) \\
& =R\left(\partial_{i}, \partial_{j}, \partial_{i}, \partial_{j}\right)+\frac{1}{\alpha^{2}}\left(g\left(\partial_{i}, \partial_{i}\right) g\left(\partial_{j}, \partial_{j}\right)\right) \\
& =2 R\left(\partial_{i}, \partial_{j}, \partial_{i}, \partial_{j}\right), \\
W_{2}\left(\partial_{i}, \partial_{j}, \partial_{j}, \partial_{i}\right) & =-W_{2}\left(\partial_{i}, \partial_{j}, \partial_{i}, \partial_{j}\right),
\end{aligned}
$$


where $i \neq j$. Direct computations show that the de Sitter space-time with metric (8) is not $W_{2}$-curvature flat. Similarly, the anti-de Sitter is not $W_{2}$-curvature flat.

- Kasner space-time in $(t, x, y, z)$ coordinates is given by

$$
d s^{2}=-d t^{2}+t^{2 \lambda_{1}} d x^{2}+t^{2 \lambda_{2}} d y^{2}+t^{2 \lambda_{3}} d z^{2},
$$

where $\lambda_{1}+\lambda_{2}+\lambda_{3}=1$ and $\lambda_{1}^{2}+\lambda_{2}^{2}+\lambda_{3}^{2}=1$. This space-time is $W_{2}$-curvature flat if $\lambda_{1}=1$.

- The Schwarzschild metric is given by

$$
d s^{2}=-\left(1-\frac{r_{s}}{r}\right) c^{2} d t^{2}+\left(\frac{1}{1-\frac{r_{s}}{r}}\right) d r^{2}+r^{2}\left(d \theta^{2}+\sin ^{2} \theta d \phi^{2}\right),
$$

where $r_{s}$ is the Schwarzchild radius and $c$ is the speed of light. The Ricci curvatures are all identically zero and so the $W_{2}$-curvature tensor is equal to the Riemann tensor.

- A cylindrically symmetric static space-time in $(t, r, \theta, \phi)$ coordinates can be given by

$$
d s^{2}=-e^{v} d t^{2}+d r^{2}+e^{v} d \theta^{2}+e^{v} d \phi^{2},
$$

where $v$ is a function of $r$. A cylindrically symmetric static space-time is $W_{2^{-}}$ curvature flat if and only if $v$ is constant. If $v$ is a nontrivial function of $r, \theta, \phi$ the situation is more complicated.

\section{1. $W_{2}$-curvature on generalized Robertson-Walker space-times}

We first define generalized Robertson-Walker space-times. Let $(M, g)$ be an $n$ dimensional Riemannian manifold and $f: I \rightarrow(0, \infty)$ be a smooth function. Then $(n+1)$-dimensional product manifold $I \times M$ furnished with the metric tensor

$$
\bar{g}=-d t^{2} \oplus f^{2} g
$$

is called a generalized Robertson-Walker space-time and is denoted by $\bar{M}=I \times_{f} M$ where $I$ is an open, connected subinterval of $\mathbb{R}$ and $d t^{2}$ is the Euclidean metric tensor on $I$. This structure was introduced to the literature to extend Robertson-Walker space-times $[17-20]$.

From now on, we will denote $\frac{\partial}{\partial t} \in \mathfrak{X}(I)$ by $\partial_{t}$ to state our results in simpler forms.

Theorem 8. Let $\bar{M}=I \times_{f} M$ be a generalized Robertson-Walker space-time equipped with the metric tensor $\bar{g}=-d t^{2} \oplus f^{2} g$. Then the curvature tensor $\bar{K}$ on $\bar{M}$ is given by

(1) $\bar{K}\left(\partial_{t}, \partial_{t}\right) \partial_{t}=\bar{K}\left(\partial_{t}, \partial_{t}\right) X=\bar{K}(X, Y) \partial_{t}=0$,

(2) $\bar{K}\left(\partial_{t}, X\right) \partial_{t}=-\frac{\ddot{f}}{f} X$,

(3) $\bar{K}\left(X, \partial_{t}\right) Y=\left[\frac{n-1}{n} g(X, Y)\left(f \ddot{f}-\dot{f}^{2}\right)-\frac{1}{n} \operatorname{Ric}(X, Y)\right] \partial_{t}$, 
(4) $\bar{K}(X, Y) Z=-R(X, Y) Z+\dot{f}^{2}[g(Y, Z) X-g(X, Z) Y]+\frac{1}{n}[\operatorname{Ric}(Y, Z) X-$ $\operatorname{Ric}(X, Z) Y]+\frac{1}{n}[g(Y, Z) X-g(X, Z) Y]\left(f \ddot{f}+(n-1) \dot{f}^{2}\right)$

for any $X, Y, Z \in \mathfrak{X}(M)$.

Now we investigate the implications of a $W_{2}$-curvature flat generalized Robertson-Walker space-time to its fiber.

Theorem 9. Let $\bar{M}=I \times_{f} M$ be a generalized Robertson-Walker space-time equipped with the metric tensor $\bar{g}=-d t^{2} \oplus f^{2} g$. Then, $\bar{M}$ is $W_{2}$-curvature flat if and only if $M$ has a constant sectional curvature $\kappa=-\dot{f}^{2}$.

Proof. Assume that $\bar{M}=I \times_{f} M$ is $W_{2}$-curvature flat, then

$$
\begin{aligned}
0= & -f \ddot{f} g(X, Y), \\
0= & \frac{1}{n} \operatorname{Ric}(X, Y)-\frac{n-1}{n} g(X, Y)\left(f \ddot{f}-\dot{f}^{2}\right), \\
0= & -f^{2} R(X, Y, Z, T)+f^{2} \dot{f}^{2}[g(Y, Z) g(X, T)-g(X, Z) g(Y, T)] \\
& +\frac{f^{2}}{n}[\operatorname{Ric}(Y, Z) g(X, T)-\operatorname{Ric}(X, Z) g(Y, T)] \\
& +\frac{f^{2}}{n}[g(Y, Z) g(X, T)-g(X, Z) g(Y, T)]\left(f \ddot{f}+(n-1) \dot{f}^{2}\right) .
\end{aligned}
$$

The first equation implies that $\ddot{f}=0$, i.e. $f=\mu t+\lambda$ and so the second equation yields

$$
\operatorname{Ric}(X, Y)=-\mu^{2}(n-1) g(X, Y) .
$$

The third equation implies that

$$
R(X, Y, Z, T)=\mu^{2}[g(Y, Z) g(X, T)-g(X, Z) g(Y, T)] .
$$

Thus the sectional curvature of $M$ is

$$
\kappa=-\mu^{2}
$$

The converse is direct by using the fact that $\bar{M}$ is Einstein with factor $(n-1) \kappa$.

A 4-dimensional space-time is called Petrov type $\mathrm{O}$ if the Weyl conformal tensor vanishes. There are many generalizations of Petrov classification for higher dimensions (see for instance [21]) but type O still has the same definition. From the above theorem, we conclude that $\bar{M}$ is flat and hence the Weyl conformal tensor vanishes.

\section{2. $W_{2}$-curvature tensor on standard static space-times}

We begin by defining standard static space-times. Let $(M, g)$ be an $n$-dimensional Riemannian manifold and $f: M \rightarrow(0, \infty)$ be a smooth function. Then 
$(n+1)$-dimensional product manifold $I \times M$ furnished with the metric tensor

$$
\bar{g}=-f^{2} d t^{2} \oplus g
$$

is called a standard static space-time and is denoted by $\bar{M}=I_{f} \times M$ where $I$ is an open, connected subinterval of $\mathbb{R}$ and $d t^{2}$ is the Euclidean metric tensor on $I$.

Note that standard static space-times can be considered as a generalization of the Einstein static universe[22-25].

Now, we are ready to study both $K$ and $W_{2}$ tensors on $\bar{M}={ }_{f} I \times M$. The following two theorems describe both tensors on $\bar{M}={ }_{f} I \times M$.

Theorem 10. Let $\bar{M}={ }_{f} I \times M$ be a standard static space-time with the metric tensor $\bar{g}=-f^{2} d t^{2} \oplus g$. If $\partial_{t} \in \mathfrak{X}(I)$ and $X, Y, Z \in \mathfrak{X}(M)$, then

(1) $\bar{K}\left(\partial_{t}, \partial_{t}\right) \partial_{t}=\bar{K}\left(\partial_{t}, \partial_{t}\right) X=\bar{K}(X, Y) \partial_{t}=0$,

(2) $\bar{K}\left(\partial_{t}, X\right) \partial_{t}=-f\left(D_{X} \nabla f+\frac{\Delta f}{n} X\right)$,

(3) $\bar{K}\left(\partial_{t}, X\right) Y=\frac{1}{n}\left(\operatorname{Ric}(X, Y)-(n+1) \frac{H^{f}(X, Y)}{f}\right) \partial_{t}$,

(4) $\bar{K}(X, Y) Z=-R(X, Y) Z+\frac{1}{n}[\operatorname{Ric}(Y, Z) X-\operatorname{Ric}(X, Z) Y]+\frac{1}{n f}\left[-H^{f}(Y, Z) X+\right.$ $\left.H^{f}(X, Z) Y\right]$.

Theorem 11. Let $\bar{M}={ }_{f} I \times M$ be a standard static space-time with the metric tensor $\bar{g}=-f^{2} d t^{2} \oplus g$. Then, $\bar{M}$ is $W_{2}$-curvature flat if and only if $M$ is flat and $H^{f}=-\frac{\Delta f}{n} g$.

Proof. Suppose that $\bar{M}={ }_{f} I \times M$ is $W_{2}$-curvature flat, then the second item of Theorem 10 implies that

$$
D_{X} \nabla f=-\frac{\Delta f}{n} X, \quad H^{f}=-\frac{\Delta f}{n} g .
$$

Taking the trace of both sides implies $\Delta f=0$ and consequently $H^{f}=0$. The third item implies that

$$
\operatorname{Ric}(X, Y)=0
$$

and so $M$ is Ricci flat. The last item of Theorem 10 implies that

$$
\begin{aligned}
& R(X, Y) Z=\frac{1}{n}[\operatorname{Ric}(Y, Z) X-\operatorname{Ric}(X, Z) Y]+\frac{1}{n f}\left[-H^{f}(Y, Z) X+H^{f}(X, Z) Y\right], \\
& R(X, Y) Z=0 .
\end{aligned}
$$

Thus $M$ is flat. The converse is straightforward.

\section{Appendix A. A Proof of Theorem 3}

Let $M=M_{1} \times{ }_{f} M_{2}$ be a warped product manifold equipped with the metric tensor $g=g_{1} \oplus f^{2} g_{2}$ where $\operatorname{dim}\left(M_{i}\right)=n_{i}, i=1,2$ and $n=n_{1}+n_{2}$. Let $X_{i}, Y_{i}, Z_{i}, T_{i} \in$ 
$\mathfrak{X}\left(M_{i}\right)$ for $i=1,2$. Then

$$
\begin{aligned}
K\left(X_{1}, Y_{1}\right) T_{1}= & -R\left(X_{1}, Y_{1}\right) T_{1}+\frac{1}{n-1}\left[\operatorname{Ric}\left(Y_{1}, T_{1}\right) X_{1}-\operatorname{Ric}\left(X_{1}, T_{1}\right) Y_{1}\right] \\
= & -R^{1}\left(X_{1}, Y_{1}\right) T_{1}+\frac{1}{n-1}\left(\operatorname{Ric}^{1}\left(Y_{1}, T_{1}\right)-\frac{n_{2}}{f} H^{f}\left(Y_{1}, T_{1}\right)\right) X_{1} \\
& -\frac{1}{n-1}\left(\operatorname{Ric}^{1}\left(X_{1}, T_{1}\right)-\frac{n_{2}}{f} H^{f}\left(X_{1}, T_{1}\right)\right) Y_{1} \\
= & -R^{1}\left(X_{1}, Y_{1}\right) T_{1}+\frac{1}{n-1}\left[\operatorname{Ric}^{1}\left(Y_{1}, T_{1}\right) X_{1}-\operatorname{Ric}^{1}\left(X_{1}, T_{1}\right) Y_{1}\right] \\
& -\frac{1}{n-1}\left[\frac{n_{2}}{f} H^{f}\left(Y_{1}, T_{1}\right) X_{1}-\frac{n_{2}}{f} H^{f}\left(X_{1}, T_{1}\right) Y_{1}\right] \\
= & K^{1}\left(X_{1}, Y_{1}\right) T_{1} \\
& -\frac{n_{2}}{(n-1)\left(n_{1}-1\right)}\left[\operatorname{Ric}^{1}\left(Y_{1}, T_{1}\right) X_{1}-\operatorname{Ric}^{1}\left(X_{1}, T_{1}\right) Y_{1}\right] \\
& -\frac{1}{n-1}\left[\frac{n_{2}}{f} H^{f}\left(Y_{1}, T_{1}\right) X_{1}-\frac{n_{2}}{f} H^{f}\left(X_{1}, T_{1}\right) Y_{1}\right] .
\end{aligned}
$$

The second case is

$$
\begin{aligned}
K\left(X_{1}, Y_{1}\right) T_{2} & =-R\left(X_{1}, Y_{1}\right) T_{2}+\frac{1}{n-1}\left[\operatorname{Ric}\left(Y_{1}, T_{2}\right) X_{1}-\operatorname{Ric}\left(X_{1}, T_{2}\right) Y_{1}\right] \\
& =0 .
\end{aligned}
$$

The third case is

$$
\begin{aligned}
K\left(X_{1}, Y_{2}\right) T_{1} & =-R\left(X_{1}, Y_{2}\right) T_{1}+\frac{1}{n-1}\left[\operatorname{Ric}\left(Y_{2}, T_{1}\right) X_{1}-\operatorname{Ric}\left(X_{1}, T_{1}\right) Y_{2}\right] \\
& =\frac{1}{f} H^{f}\left(X_{1}, T_{1}\right) Y_{2}-\frac{1}{n-1} \operatorname{Ric}^{1}\left(X_{1}, T_{1}\right) Y_{2}+\frac{n_{2}}{(n-1) f} H^{f}\left(X_{1}, T_{1}\right) Y_{2} \\
& =-\left[\frac{1}{n-1} \operatorname{Ric}^{1}\left(X_{1}, T_{1}\right)-\frac{n+n_{2}-1}{(n-1) f} H^{f}\left(X_{1}, T_{1}\right)\right] Y_{2} .
\end{aligned}
$$

The next case is

$$
\begin{aligned}
K\left(X_{1}, Y_{2}\right) T_{2}= & -R\left(X_{1}, Y_{2}\right) T_{2}+\frac{1}{n-1}\left[\operatorname{Ric}\left(Y_{2}, T_{2}\right) X_{1}-\operatorname{Ric}\left(X_{1}, T_{2}\right) Y_{2}\right] \\
= & -f g_{2}\left(Y_{2}, T_{2}\right) D_{X_{1}}^{1} \nabla f+\frac{1}{n-1} \operatorname{Ric}^{2}\left(Y_{2}, T_{2}\right) X_{1} \\
& -\frac{f^{\sharp}}{n-1} g_{2}\left(Y_{2}, T_{2}\right) X_{1} .
\end{aligned}
$$

Also,

$$
\begin{aligned}
K\left(X_{2}, Y_{2}\right) T_{1} & =-R\left(X_{2}, Y_{2}\right) T_{1}+\frac{1}{n-1}\left[\operatorname{Ric}\left(Y_{2}, T_{1}\right) X_{2}-\operatorname{Ric}\left(X_{2}, T_{1}\right) Y_{2}\right] \\
& =0
\end{aligned}
$$


Finally,

$$
\begin{aligned}
K\left(X_{2}, Y_{2}\right) T_{2}= & -R\left(X_{2}, Y_{2}\right) T_{2}+\frac{1}{n-1}\left[\operatorname{Ric}\left(Y_{2}, T_{2}\right) X_{2}-\operatorname{Ric}\left(X_{2}, T_{2}\right) Y_{2}\right] \\
= & -R^{2}\left(X_{2}, Y_{2}\right) T_{2}+\|\nabla f\|_{1}^{2}\left[g_{2}\left(X_{2}, T_{2}\right) Y_{2}-g_{2}\left(Y_{2}, T_{2}\right) X_{2}\right] \\
& +\frac{1}{n-1}\left[\operatorname{Ric}^{2}\left(Y_{2}, T_{2}\right)-f^{\sharp} g_{2}\left(Y_{2}, T_{2}\right)\right] X_{2} \\
& -\frac{1}{n-1}\left[\operatorname{Ric}^{2}\left(X_{2}, T_{2}\right)-f^{\sharp} g_{2}\left(X_{2}, T_{2}\right)\right] Y_{2} .
\end{aligned}
$$

Then

$$
\begin{aligned}
K\left(X_{2}, Y_{2}\right) T_{2}= & -R^{2}\left(X_{2}, Y_{2}\right) T_{2}+\frac{1}{n-1} \operatorname{Ric}^{2}\left(Y_{2}, T_{2}\right) X_{2}-\frac{1}{n-1} \operatorname{Ric}^{2}\left(X_{2}, T_{2}\right) Y_{2} \\
& +\|\nabla f\|_{1}^{2}\left[g_{2}\left(X_{2}, T_{2}\right) Y_{2}-g_{2}\left(Y_{2}, T_{2}\right) X_{2}\right] \\
& -\frac{f^{\sharp}}{n-1}\left(g_{2}\left(Y_{2}, T_{2}\right) X_{2}-g_{2}\left(X_{2}, T_{2}\right) Y_{2}\right)
\end{aligned}
$$

and so

$$
\begin{aligned}
K\left(X_{2}, Y_{2}\right) T_{2}= & -R^{2}\left(X_{2}, Y_{2}\right) T_{2}+\frac{1}{n-1}\left[\operatorname{Ric}^{2}\left(Y_{2}, T_{2}\right) X_{2}-\operatorname{Ric}^{2}\left(X_{2}, T_{2}\right) Y_{2}\right] \\
& -\left(\|\nabla f\|_{1}^{2}+\frac{f^{\sharp}}{n-1}\right)\left[g_{2}\left(Y_{2}, T_{2}\right) X_{2}-g_{2}\left(X_{2}, T_{2}\right) Y_{2}\right] \\
= & -R^{2}\left(X_{2}, Y_{2}\right) T_{2}+\frac{1}{n-1}\left[\operatorname{Ric}^{2}\left(Y_{2}, T_{2}\right) X_{2}-\operatorname{Ric}^{2}\left(X_{2}, T_{2}\right) Y_{2}\right] \\
& +\left(\|\nabla f\|_{1}^{2}+\frac{f^{\sharp}}{n-1}\right)\left[g_{2}\left(X_{2}, T_{2}\right) Y_{2}-g_{2}\left(Y_{2}, T_{2}\right) X_{2}\right] .
\end{aligned}
$$

Thus

$$
\begin{aligned}
& K\left(X_{2}, Y_{2}\right) T_{2} \\
&= K^{2}\left(X_{2}, Y_{2}\right) T_{2}-\frac{n_{1}}{(n-1)\left(n_{2}-1\right)}\left[\operatorname{Ric}^{2}\left(Y_{2}, T_{2}\right) X_{2}-\operatorname{Ric}^{2}\left(X_{2}, T_{2}\right) Y_{2}\right] \\
&+\left(\|\nabla f\|_{1}^{2}+\frac{f^{\sharp}}{n-1}\right)\left[g_{2}\left(X_{2}, T_{2}\right) Y_{2}-g_{2}\left(Y_{2}, T_{2}\right) X_{2}\right]
\end{aligned}
$$

and the proof is now complete.

\section{Acknowledgment}

We would like to thank the referee for the careful review and the valuable comments, which provided insights that helped us to improve the quality of the paper.

\section{References}

[1] G. P. Pokhariyal and R. S. Mishra, Curvature tensors and their relativistic significance, Yokohama Math. J. 18 (1970) 105-108. 
[2] S. Mallick and U. D. De, Space-times admitting $W_{2}$-curvature tensor, Int. J. Geom. Meth. Mod. Phys. 11(4) (2014) 1450030.

[3] G. P. Pokhariyal, Relativistic significance of curvature tensors, Int. J. Math. Math. Sci. 5(1) (1982) 133-139.

[4] G. P. Pokhariyal, Curvature tensors on A-Einstein Sasakian manifolds, Balkan J. Geom. Appl. 6(1) (2001) 45-50.

[5] F. O. Zengin, On Riemannian manifolds admitting $W_{2}$-curvature, Miskolc Math. Notes 12(2) (2011) 289-296.

[6] A. A. Shaikh, Y. Matsuyama and S. K. Jana, On a type of general relativistic spacetime with $W_{2}$-curvature tensor, Indian J. Math. 50(1) (2008) 53-62.

[7] S. K. Hui and R. S. Lemence, On generalized quasi-Einstein manifold admitting $W_{2}$-curvature tensor, Int. J. Math. Anal. 6(21-24) (2012) 1115-1121.

[8] S. K. Hui and A. Sarkar, On the $W_{2}$-curvature tensor of generalized Sasakian-spaceforms, Math. Pannon. 23(1) (2012) 113-124.

[9] R. N. Singh and G. Pandey, On the $W_{2}$-curvature tensor of the semi-symmetric nonmetric connection in a Kenmotsu manifold, Novi Sad J. Math. 43(2) (2013) 91-105.

[10] R. N. Singh, S. K. Pandey and G. Pandey, On $W_{2}$-curvature tensor in a Kenmotsu manifold, Tamsui Oxf. J. Inf. Math. Sci. 29(2) (2013) 129-141.

[11] R. L. Bishop and B. O'Neill, Manifolds of negative curvature, Trans. Amer. Math. Soc. 145 (1969) 1-49.

[12] B. O'Neill, Semi-Riemannian Geometry with Applications to Relativity (Academic Press, London, 1983).

[13] H. Shima, The Geometry of Hessian Structures (World Scientific, New Jersey, 2007).

[14] H. Shima and K. Yagi, Geometry of Hessian manifolds, Differential. Geom. Appl. 7(3) (1997) 277-290.

[15] G. Bercu, Pseudo-Riemannian structures with the same connection, Balkan J. Geom. Appl. 11(1) (2006) 23-38.

[16] A. Salimov and A. Gezer, Norden structures of Hessian type, Turkish. J. Math. 38(3) (2014) 462-469.

[17] S. Shenawy and B. Unal, 2-Killing vector fields on warped product manifolds, Int. J. Math. 26 (2015) 17 pp.

[18] J. L. Flores and M. Sánchez, Geodesic connectedness and conjugate points in GRW space-times, J. Geom. Phys. 36(3-4) (2000) 285-314.

[19] M. Sánchez, On the geometry of generalized Robertson-Walker spacetimes: Curvature and Killing fields, J. Geom. Phys. 31(1) (1999) 1-15.

[20] M. Sánchez, On the geometry of generalized Robertson-Walker spacetimes: Geodesics, Gen. Relativ. Gravit. 30(6) (1998) 915-932.

[21] A. Coley, R. Milson, V. Pravda and A. Pravdová, Classification of the Weyl tensor in higher dimensions, Class. Quantum Grav. 21 (2004) L35-L41.

[22] D. E. Allison, Energy conditions in standard static space-times, Gen. Relativ. Gravit. 20(2) (1998) 115-122.

[23] D. E. Allison, Geodesic completeness in static space-times, Geom. Dedicata 26 (1988) 85-97.

[24] D. E. Allison and B. Ünal, Geodesic structure of standard static space-times, J. Geom. Phys. 46(2) (2003) 193-200.

[25] A. L. Besse, Einstein Manifolds, Classics in Mathematics (Springer-Verlag, Berlin, 2008). 